\title{
BEST DIOPHANTINE APPROXIMATIONS TO A SET OF LINEAR FORMS
}

\author{
J. C. LAGARIAS \\ (Received 4 May 1981; revised 23 February 1982) \\ Communicated by A. J. van der Poorten
}

\begin{abstract}
We define the notion of a best Diophantine approximation vector to a set of linear forms. This generalizes definitions of a best approximation vector to a single linear form and of a best simultaneous Diophantine approximation vector. We derive necessary and sufficient conditions for the existence of an infinite set of best Diophantine approximation vectors. Finally, we prove that such approximation vectors are spaced far apart in an appropriate sense.
\end{abstract}

1980 Mathematics subject classification (Amer. Math. Soc.): 10 F 10.

\section{Introduction}

This paper defines the notion of a best Diophantine approximation vector to a set of linear forms. This definition generalizes the notions of a best approximation to a single linear form in [1], [2], [4] and of a best simultaneous Diophantine approximation given in [3], [5], [6], [7]. We derive necessary and sufficient conditions for an infinite set of best Diophantine approximation vectors to exist. Finally we prove that best approximation vectors are spaced far apart, in an appropriate sense.

Let $\left\{l_{i}\right\}_{i=1}^{n}$ be a set of $n$ linear forms in $m$ variables and let $L: \mathbf{R}^{m} \rightarrow \mathbf{R}^{n}$ be the linear transformation defined by

$$
L\left(\left(x_{1}, \ldots, x_{m}\right)\right)=\left(l_{1}, l_{2}, \ldots, l_{n}\right)
$$

where

$$
l_{i}=\alpha_{1}^{(i)} x_{1}+\cdots+\alpha_{n}^{(i)} x_{m}
$$

C. Copyright Australian Mathematical Society 1983 
for $1 \leqslant i \leqslant n$. A seminorm $\|\cdot\|$ on $\mathbf{R}^{k}$ is a map $\mathbf{R}^{k} \rightarrow \mathbf{R}$ satisfying the axioms

(i) $\|\mathbf{x}\| \geqslant 0$

(ii) $\|a \mathbf{x}\|=|a|\|\mathbf{x}\|$ for $a \in \mathbf{R}$ and $\mathbf{x} \in \mathbf{R}^{k}$,

(iii) $\left\|\mathbf{x}_{1}+\mathbf{x}_{2}\right\| \leqslant\left\|\mathbf{x}_{1}\right\|+\left\|\mathbf{x}_{2}\right\|$ for $\mathbf{x}_{1}, \mathbf{x}_{2} \in \mathbf{R}^{k}$.

The basic definition is as follows.

Definition. A nonzero integer vector $\mathbf{v} \in \mathbf{Z}^{m}$ is a best (Diophantine) approximation vector to $L$ with respect to the seminorms $\|\cdot\|_{1}$ on $\mathbf{R}^{m}$ and $\|\cdot\|_{2}$ on $\mathbf{R}^{n}$ provided that for all $\mathbf{w} \in \mathbf{Z}^{n}$ the following implications hold.

$$
\begin{aligned}
& \text { (1) }\|\mathbf{w}\|_{1} \leqslant\|\mathbf{v}\|_{1} \Rightarrow\|L(\mathbf{w})\|_{2} \geqslant\|L(\mathbf{v})\|_{2} . \\
& \text { (2) }\|L(\mathbf{w})\|_{2} \leqslant\|L(\mathbf{v})\|_{2} \Rightarrow\|\mathbf{w}\|_{1} \geqslant\|\mathbf{v}\|_{1} .
\end{aligned}
$$

(Note that (1) and (2) are equivalent except for cases of equality.)

A best approximation vector $\mathbf{v}$ is nontrivial if $\|\mathbf{v}\|_{1} \neq 0$ and $\|L(\mathbf{v})\|_{2} \neq 0$. If for any $\varepsilon>0$ it is possible to find $\mathbf{v} \in \mathbf{Z}^{m}$ (depending on $\varepsilon$ ) such that

$$
\begin{aligned}
& 0<\|\mathbf{v}\|_{1}<\varepsilon, \\
& 0<\|L(\mathbf{v})\|_{2}<\varepsilon,
\end{aligned}
$$

then no nontrivial best approximation vectors can exist. We will show that this possibility is excluded if $\|\cdot\|_{1},\|\cdot\|_{2}$ and $L$ satisfy the transversality condition defined below. Any seminorm $\|\cdot\|$ on $\mathbf{R}^{k}$ has a zero set given by

$$
V=V(\|\cdot\|)=\{\mathbf{x}:\|\mathbf{x}\|=0\},
$$

which is a subspace of $\mathbf{R}^{k}$. Let $V_{1}, V_{2}$ denote the zero sets of $\|\cdot\|_{1},\|\cdot\|_{2}$ respectively.

Definition. $L$ is transverse with respect to $\|\cdot\|_{1}$ and $\|\cdot\|_{2}$ if

$$
V_{1} \cap L^{-1}\left(V_{2}\right)=\{\mathbf{0}\} \text {. }
$$

We also make the following definitions which are relevant in determining the order type of the set of best approximation vectors.

Definition. $\|\cdot\|_{1}$ is collapsed if there is a nonzero $\mathbf{x} \in \mathbf{Z}^{m}$ with $\|\mathbf{x}\|_{1}=0$.

Definition. $L$ is degenerate with respect to $\|\cdot\|_{2}$ if there exists a nonzero $\mathbf{v} \in \mathbf{Z}^{m}$ such that $\|L(\mathbf{v})\|_{2}=0$. Otherwise $L$ is nondegenerate.

The following result characterizes the existence of best approximation vectors.

THEOREM 1.1. If $L$ is not transverse with respect to $\|\cdot\|_{1}$ and $\|\cdot\|_{2}$ then there are no nontrivial best approximation vectors. If $L$ is transverse with respect to $\|\cdot\|_{1}$ and $\|\cdot\|_{2}$ then $L$ has a set $B$ of nontrivial best approximation vectors which can be numbered $\left\{\mathbf{v}_{j}\right\}$ for $n_{0}<j<n_{1}$ such that for all $n_{0}<j<k<n_{1}$,

$$
\left\|\mathbf{v}_{j}\right\|_{1} \leqslant\left\|\mathbf{v}_{k}\right\|_{1} \text { and }\left\|L\left(\mathbf{v}_{j}\right)\right\|_{2} \geqslant\left\|L\left(\mathbf{v}_{k}\right)\right\|_{2} .
$$


In addition:

(1) $n_{0}=-\infty$ if and only if $\|\cdot\|_{1}$ is not collapsed and $\|\cdot\|_{1}$ is not a norm.

(2) $n_{1}=+\infty$ if and only if $L$ is nondegenerate and either $\|\cdot\|_{2}$ is not a norm or

$\|\cdot\|_{2}$ is a norm and $L$ does not have full row rank.

If $n_{1}=+\infty$ then $\left\|\mathbf{v}_{n}\right\|_{1} \rightarrow \infty$ and $\left\|L\left(\mathbf{v}_{n}\right)\right\|_{2} \rightarrow 0$ as $n \rightarrow \infty$.

The following result concerns the spacing of best approximation vectors.

THEOREM 1.2. Let $L$ be transverse with respect to $\|\cdot\|_{1}$ and $\|\cdot\|_{2}$. Let $\left\{\mathbf{v}_{j}\right\}$ be the set of best approximation vectors. If $D=7^{m}$ then

$$
\left\|\mathbf{v}_{k+D}\right\|_{1} \geqslant 2\left\|\mathbf{v}_{k}\right\|_{1}
$$

holds for all $k$ for which $\mathbf{v}_{k+D}, \mathbf{v}_{k}$ exist.

Theorems 1.1 and 1.2 are proved in Sections 2 and 3 respectively. Before giving the proofs we indicate how the notions of best approximation in [1] - [7] are special cases of the definition given here.

EXAMPLE 1 (Approximations to a Single Linear Form). Let

$$
L: \mathbf{R}^{n+1} \rightarrow \mathbf{R} \text { via } l_{i}=\alpha_{1} x_{1}+\cdots+\alpha_{n} x_{n}-x_{0} .
$$

Given a norm $\|\cdot\|$ on $\mathbf{R}^{n}$, let $\|\cdot\|$ be the seminorm on $\mathbf{R}^{n+1}$ defined by $\left\|\left(x_{0}, x_{1}, \ldots, x_{n}\right)\right\|_{1}=\left\|\left(x_{1}, \ldots, x_{n}\right)\right\| .\|\cdot\|_{2}$ is taken to be the sup norm on $\mathbf{R}^{1}$. Note that $\|\cdot\|_{1}$ is collapsed. $L$ is always transverse and is nondegenerate if and only if

$$
\operatorname{dim}_{\mathbf{Q}}\left[1, \alpha_{1}, \ldots, \alpha_{n}\right]=n+1
$$

EXAMPLE 2 (Best simultaneous Diophantine approximations). Let

$$
L: \mathbf{R}^{n+1} \rightarrow \mathbf{R}^{n} \quad \text { via } \quad l_{i}=\alpha_{i} x_{0}-x_{i}
$$

for $1 \leqslant i \leqslant n$. Here $\|\cdot\|_{1}$ is the seminorm

$$
\|\mathbf{x}\|_{1}=\left|x_{0}\right|
$$

and $\|\cdot\|_{2}=\|\cdot\|$ is an arbitrary norm on $\mathbf{R}^{n}$. Note $\|\cdot\|_{1}$ is collapsed and $L$ is always transverse. $L$ is nondegenerate exactly when some $\alpha_{i} \notin \mathbf{Q}$.

In both examples, Theorem 1.1 guarantees that if $L$ is nondegenerate then the set $B$ of best approximations can be written $\left\{\mathbf{v}_{k}\right\}$ for $1 \leqslant k<\infty$ such that (1.8) holds. 


\section{Existence of best approximation vectors}

Proof of Theorem 1.1. Suppose $L$ is not transverse, so that $V_{1} \cap L^{-1}\left(V_{2}\right)=$ $V_{3} \neq \mathbf{0}$. Let $\|\cdot\|_{e}$ denote the Euclidean norm on $\mathbf{R}^{m}$ and $\pi$ orthogonal projection onto $V_{3}$. For any $\varepsilon>0$ we can find a nonzero $\mathbf{v} \in \mathbf{Z}^{m}$ with

$$
\|\mathbf{v}-\pi(\mathbf{v})\|_{e}<\varepsilon .
$$

If $A=\max \left|\alpha_{j}^{(i)}\right|$, where the $\alpha_{j}^{(i)}$ are given by (1.2), then

$$
\|L(\mathbf{v})-L(\pi(\mathbf{v}))\|_{e}<A \varepsilon .
$$

Now there exist positive constants $c_{1}, c_{2}$ such that

$$
c_{1}\|\mathbf{x}\|_{e} \geqslant\|\mathbf{x}\|_{1}, \quad c_{2}\|\mathbf{y}\|_{e} \geqslant\|\mathbf{y}\|_{2},
$$

for all $\mathbf{x} \in \mathbf{R}^{m}, \mathbf{y} \in \mathbf{R}^{n}$, where

$$
c_{i}=\sup \left\{\|\mathbf{x}\|_{i}:\|\mathbf{x}\|_{e}=1\right\} \text { for } i=1,2 .
$$

Hence

$$
\begin{aligned}
\|\mathbf{v}\|_{1} & =\|\mathbf{v}-\pi(\mathbf{v})\|_{1}<c_{1} \varepsilon, \\
\|L(\mathbf{v})\|_{2} & =\|L(\mathbf{v})-L(\pi(\mathbf{v}))\|_{2}<c_{2} A \varepsilon .
\end{aligned}
$$

Since $\varepsilon$ can be chosen arbitrarily small, there are no best approximation vectors with $\|\mathbf{x}\|_{1} \neq 0,\|L(\mathbf{x})\|_{2} \neq 0$.

Now suppose $L$ is transverse. Consider the $m$-dimensional subspace

$$
W=\left\{(\mathbf{x}, L(\mathbf{x})): \mathbf{x} \in \mathbf{R}^{m}\right\}
$$

of $\mathbf{R}^{m+n}$. For $\mathbf{x} \in \mathbf{R}^{m}$ we set $\mathbf{x}^{*}=(\mathbf{x}, L(\mathbf{x})) \in W$. The map $\mathbf{x} \rightarrow \mathbf{x}^{*}$ is a linear vector space isomorphism. Define a seminorm $\|\cdot\|$ on $W$ by

$$
\left\|\mathbf{x}^{*}\right\|=\|\mathbf{x}\|_{1}+\|L(\mathbf{x})\|_{2} .
$$

Then $\|\cdot\|$ is a norm by the transversality condition. Now set

$$
\Omega\left(c_{1}, c_{2}\right)=\left\{\mathbf{x}^{*} \in W:\|\mathbf{x}\|_{1} \leqslant c_{1} \text { and }\|L(\mathbf{x})\|_{2} \leqslant c_{2}\right\} .
$$

This is a centrally symmetric convex body. We define a volume on $W$ arising from $m$-dimensional Lebesgue measure on $\mathbf{R}^{m}$ via the map $\mathbf{x} \rightarrow \mathbf{x}^{*}$. Then

$$
\Lambda=\left\{(\mathbf{x}, L(\mathbf{x})): \mathbf{x} \in \mathbf{Z}^{m}\right\}
$$

is a lattice in $W$ whose unit cell has volume 1 . Let

$$
B(c)=\left\{\mathbf{x}^{*} \in W:\left\|\mathbf{x}^{*}\right\| \leqslant c\right\},
$$

and observe that

$$
B(c) \subseteq \Omega(c, c) \subseteq B(2 c) .
$$

For sufficiently small $c, B(2 c)$ and hence $\Omega(c, c)$ contains only the point $\mathbf{0}$ of $\Lambda$ since $\|\cdot\|$ is a norm. By Minkowski's theorem, $\Omega(c, c)$ contains a nonzero lattice 
point if its volume exceeds $2^{m}$, which must occur for some $c$ by (2.3). Now take the smallest $c$ for which $\Omega(c, c)$ has points of $\Lambda$ on its boundary but none except 0 inside. The finite set $B_{0}^{*}$ of lattice points $\mathbf{x}^{*}$ on the boundary can be divided into three classes, those with

(1) $\|\mathbf{x}\|_{1}<c,\|L(\mathbf{x})\|_{2}=c$,

(2) $\|\mathbf{x}\|_{1}=c,\|L(\mathbf{x})\|_{2}<c$,

(3) $\|\mathbf{x}\|_{1}=\|L(\mathbf{x})\|_{2}=c$.

If there are vectors $\mathbf{x}$ satisfying (1), those $\mathbf{x}$ with the smallest value of $\|\mathbf{x}\|_{1}$ are best approximation vectors. Call this set of vectors $B_{-1}$. If there are vectors $x$ satisfying (2), those $\mathbf{x}$ with the smallest value of $\|L(\mathbf{x})\|_{2}$ are best approximation vectors. Call this set of vectors $B_{1}$. All vectors satisfying (3) are best approximation vectors if there are no $\mathbf{x}$ satisfying either (1) or (2), otherwise none are. Call this set of vectors $B_{0}$. Note that at least one of $B_{-1}, B_{0}, B_{1}$ is empty. Let $\left(d_{-1}, r_{-1}\right)$, $\left(d_{0}, r_{0}\right)$ and $\left(d_{1}, r_{1}\right)$ denote the values of $\left(\|\mathbf{x}\|_{1},\|L(\mathbf{x})\|_{2}\right)$ in each of the sets $B_{-1}$, $B_{0}, B_{1}$, respectively, which are nonempty, and set them equal to $(c, c)$ otherwise.

Now start with $\Omega\left(c, r_{1}\right)$ with $c=d_{1}$ and increase $c$ until the first value $d_{2}$ is encountered at which an element of $\Lambda$ with $\|L(\mathbf{x})\|_{2}<r_{1}$ appears on the boundary of $\Omega\left(d_{2}, r_{1}\right)$. Let $B_{2}$ be the set of lattice points on this boundary with minimal $\|L(\mathbf{x})\|_{2}=r_{2}$. The elements of $B_{2}$ are all best approximation vectors. Now repeat this process starting with $\Omega\left(c, r_{2}\right)$ with $c=d_{2}$, and continue it to construct $B_{3}, B_{4}, \ldots$ If for some $\Omega\left(c, r_{j}\right)$ we can let $c \rightarrow \infty$ with no nonzero element of $\Lambda$ ever appearing on the boundary having $\|L(\mathbf{x})\|_{2}<r_{j}$, we say this process terminates on the right at $B_{j}$.

Now start with $\Omega\left(d_{-1}, c\right)$ with $c=r_{-1}$ and increase $c$ until the first value $r_{-2}$ is encountered at which $\|\mathbf{x}\|_{1}<d_{-1}$. Let $B_{-2}$ be the set of vectors on the boundary of $\Omega\left(d_{-2}, r_{-2}\right)$ with minimal $\|\mathbf{x}\|_{1}=d_{-2}$. Now repeat this process starting with $\Omega\left(d_{-2}, c\right)$ with $c=r_{-2}$ and continue it to construct $B_{-2}, B_{-3}, \ldots$ If for some $\Omega\left(d_{-j}, c\right)$ we can let $c \rightarrow \infty$ with no nonzero element of $\Lambda$ ever appearing with $\|\mathbf{x}\|_{1}<d_{-j}$, we say this process terminates on the left at $B_{-j}$.

We obtain in this way a sequence $\left\{\left(d_{j}, r_{j}\right):-n_{1}<j<n_{2}\right\}$ in which $d_{j-1}<d_{j}$ (except for $j=0,1$ where equality may occur) and $r_{j-1}>r_{j}$ (except for $j=0,1$ where equality may occur). Note that this construction shows there are no best approximation vectors with $d_{j-1}<\|\mathbf{x}\|_{1}<d_{j}$ and produces all those with $\|\mathbf{x}\|=$ $d_{j}$; also that there are none with $r_{j-1}>\|L(\mathbf{x})\|_{2}>r_{j}$ and it produces all those with $\|L(\mathbf{x})\|_{2}=r_{j}$. If truncation on the left occurs at $\left(d_{-j}, r_{-j}\right)$, this construction guarantees there are no best approximation vectors with $0<\|\mathbf{x}\|_{1}<d_{-j}$ or with $\|L(\mathbf{x})\|_{2}>r_{j}$. If truncation on the right occurs, it guarantees there are no best approximation vectors with $0 \leqslant\|L(\mathbf{x})\|_{2}<r_{-j}$, or with $\|\mathbf{x}\|_{1}>d_{j}$. Thus the construction produces all best approximation vectors $\mathbf{x}$ with

$$
\lim _{k \rightarrow \infty} d_{-k}<\|\mathbf{x}\|_{1}<\lim _{k \rightarrow \infty} d_{k}
$$


and

$$
\lim _{k \rightarrow \infty} r_{-k}>\|L(\mathbf{x})\|_{2}>\lim _{k \rightarrow \infty} r_{k},
$$

where we use the convention that if termination on the left occurs then $\lim _{k \rightarrow \infty} d_{-k}=0, \lim _{k \rightarrow \infty} r_{-k}=\infty$, and if termination on the right. occurs, then $\lim _{k \rightarrow \infty} d_{k}=\infty, \lim _{k \rightarrow \infty} r_{k}=0$.

This process of obtaining the best approximations is geometrically similar to that of Voronoi's algorithm for finding units in a non-totally real cubic field (for example, see [8]).

We next check that the set of elements in the union of the $B_{j}$ 's exhausts the set $B$ of best approximation vectors. Suppose not, so that there is such a best approximation vector $\mathbf{x}$. By the remarks above, it must be either that termination on the right doesn't occur and that

$$
\|\mathbf{x}\|_{1} \geqslant \lim _{j \rightarrow \infty} d_{j}, \quad\|L(\mathbf{x})\|_{2} \leqslant \lim _{j \rightarrow \infty} r_{j},
$$

or else that termination on the left doesn't occur and

$$
\|\mathbf{x}\|_{1} \leqslant \lim _{j \rightarrow \infty} d_{-j}, \quad\|L(\mathbf{x})\|_{2} \geqslant \lim _{j \rightarrow \infty} r_{-j} .
$$

We will rule out both these possibilities by showing that if termination on the right doesn't occur, then $d_{j} \rightarrow \infty$ and $r_{j} \rightarrow 0$ as $j \rightarrow \infty$, and that if termination on the left doesn't occur, then $d_{-j} \rightarrow 0, r_{-j} \rightarrow \infty$ as $j \rightarrow \infty$.

We treat the case that termination on the right doesn't occur. Pick an element $\mathbf{x}_{j} \in B_{j}$ for all $j \geqslant 2$. Then $\left\|L\left(\mathbf{x}_{j}\right)\right\|_{2}=r_{j}$ and $r_{2}>r_{3}>r_{4}>\cdots$. Now $\left\|\left(\mathbf{x}_{j}, L\left(\mathbf{x}_{j}\right)\right)\right\|=r_{j}+d_{j} \rightarrow \infty$ as $j \rightarrow \infty$ since $\|\cdot\|$ is a norm and $\mathbf{x}_{j}^{*} \in \Lambda$. Since $r_{j}$ is bounded, $d_{j} \rightarrow \infty$ as $j \rightarrow \infty$. It remains to show $r_{j} \rightarrow 0$ as $j \rightarrow \infty$. To do this it suffices to prove the following fact.

FACT. If termination on the right doesn't occur, then for any $\varepsilon>0$ there exists a nonzero $\mathbf{x} \in \mathbf{Z}^{m}$ such that $\|L(\mathbf{x})\|_{2}<\boldsymbol{\varepsilon}$.

If the Fact is true, we can find some $\mathbf{x}_{j} \in B_{j}$ for which $\left\|\mathbf{x}_{j}\right\|_{1}>\|\mathbf{x}\|_{1}$, in which case by the definition of best approximation vector $r_{j}=\left\|L\left(\mathbf{x}_{j}\right)\right\|_{2} \leqslant\|L(\mathbf{x})\|_{2}<\varepsilon$. Hence $r_{j} \rightarrow 0$ as required.

To prove the Fact, we observe that its conclusion is equivalent to the statement that for any positive $\varepsilon$ the region $\Omega(c, \varepsilon)$ contains a nonzero point of $\Lambda$, for some sufficiently large $c=c(\varepsilon)$. This occurs if:

1. $\|\cdot\|_{2}$ is not a norm. In this case $L^{-1}(\mathbf{0})$ is a non-trivial subspace of $\mathbf{R}^{m}$. Then the set $\left\{\mathbf{x}:\|L(\mathbf{x})\|_{2}<\mathbf{\varepsilon}\right\}$ contains a small open ball around the origin translated by all elements of $L^{-1}(\mathbf{0})$, hence has infinite volume in $\mathbf{R}^{m}$. Consequently the 
volume of $\Omega(c, \varepsilon)$ is unbounded as $c \rightarrow \infty$, and so contains nonzero lattice points of $\Lambda$ for sufficiently large $c$ by Minkowski's theorem.

2. $L$ does not have full rank $m$. Then $L\left(\mathbf{Z}^{m}\right)$ projects onto a $k$-dimensional subspace $\mathbf{S}$ of $\mathbf{R}^{n}$, where $k \leqslant m-1$. A standard pigeonhole principle argument then proves for any $\varepsilon$ there exist nonzero $\mathbf{x} \in \mathbf{Z}^{m}$ with $\|L(\mathbf{x})\|_{2}<\varepsilon$.

The exceptional case is where $\|\cdot\|_{2}$ is a norm and $L$ has full rank $m$. In this case $L\left(\mathbf{Z}^{m}\right)$ is a lattice, and for any $c_{0}$ there are only a finite number of $\mathbf{x} \in \mathbf{Z}^{m}$ such that $\|L(\mathbf{x})\|_{2}<c_{0}$, so termination on the right occurs in this case. This proves the fact.

One shows that $d_{j} \rightarrow 0$ and $r_{j} \rightarrow \infty$ as $j \rightarrow \infty$ if termination on the left does not occur by similar arguments.

We obtain a numbering $\left\{\mathbf{v}_{j}\right\}$ of the elements of $B$ that satisfies (1.8) by ordering the elements of each $B_{j}$ in a way that satisfies (1.8), concatenating the sets $B_{j}$ using the obvious ordering, and picking $\mathbf{v}_{0} \in B_{0}$.

It remains to specify the conditions under which termination on the left or right occurs.

If $\|\cdot\|_{1}$ is not a norm, then $\Omega\left(c_{1}, c_{2}\right)$ has unbounded volume as $c_{2} \rightarrow \infty$. Minkowski's theorem then asserts that for large enough $c_{2}$ there must exist nonzero lattice points with $\|\mathbf{x}\|_{1}<c_{1},\|L(\mathbf{x})\|_{2}<c_{2}$. Thus termination on the left can occur only if there is a nonzero $\mathbf{x} \in \mathbf{Z}^{m}$ with $\|\mathbf{x}\|_{1}=0$, that is, $\|\cdot\|_{1}$ is collapsed. If $\|\cdot\|_{1}$ is a norm there exists $c_{0}$ so that $\|\mathbf{x}\|_{1}<c_{0}$ contains only $\mathbf{0} \in \mathbf{Z}^{m}$ and termination on the left occurs.

If $\|\cdot\|_{2}$ is not a norm then $\Omega\left(c_{1}, c_{2}\right)$ has unbounded volume as $c_{1} \rightarrow \infty$. As above, lattice points with $\|\mathbf{x}\|_{1}<c_{1},\|L(\mathbf{x})\|_{2}<c_{2}$ must occur. In this case termination on the right can occur only if there is an $\mathbf{x}$ with $\|L(\mathbf{x})\|_{2}=0$, that is, $L$ is degenerate with respect to $\|\cdot\|_{2}$. If $\|\cdot\|_{2}$ is a norm and $L$ has rank $m$, then it was shown earlier that termination on the right occurs. If $L$ has rank $<m$, then $\|L(\mathbf{x})\|_{2}<\varepsilon$ has an infinite number of solutions, and termination on the right can occur only if $L$ is degenerate with respect to $\|\cdot\|_{2}$.

\section{Spacing of best approximation vectors}

Proof of Theorem 1.2. Let $D=7^{m}$, and set $\left\|\mathbf{v}_{k}\right\|_{1}=r_{0},\left\|L\left(\mathbf{v}_{k}\right)\right\|_{2}=r_{1}$. Suppose that

$$
\left\|\mathbf{v}_{k+d}\right\|_{1} \leqslant 2\left\|\mathbf{v}_{k}\right\|_{1} .
$$

By definition of a best approximation vector, we know that for $\mathbf{w} \neq 0$ in $\mathbf{Z}^{m}$,

$$
\|\mathbf{w}\|_{1} \leqslant r_{0} \Rightarrow\left\|L\left(\mathbf{w}_{1}\right)\right\|_{2} \geqslant r_{1} .
$$


Now consider the seminorm $\|\cdot\|_{c}$ defined on $W$ by

$$
\|(\mathbf{x}, L(\mathbf{x}))\|_{c}=\|\mathbf{x}\|_{1}+c\|L(\mathbf{x})\|_{2}
$$

where $c>0$. Then $\|\cdot\|_{c}$ is actually a norm on $W$ by the transversality condition. For $\mathbf{x} \in \mathbf{R}^{m}$ let $\mathbf{x}^{*}=(\mathbf{x}, L(\mathbf{x})) \in W$. We choose $c=r_{0} / r_{1}$ so that

$$
\left\|\mathbf{v}_{k}^{*}\right\|_{c}=2 r_{0} \text {. }
$$

Since $\left\|L\left(\mathbf{v}_{k+i}\right)\right\|_{2} \leqslant\left\|L\left(\mathbf{v}_{k}\right)\right\|_{2}$, (3.1) yields, for $0 \leqslant i \leqslant D$,

$$
\left\|\mathbf{v}_{k+i}^{*}\right\|_{c} \leqslant 3 r_{0} .
$$

Now for $0 \leqslant i<j \leqslant D$,

$$
\mathbf{w}=\mathbf{v}_{k+j}-\mathbf{v}_{k+i} \neq \mathbf{0} .
$$

Using (3.2) we obtain that either $\|\mathbf{w}\|_{1}>r_{0}$ or $\|L(\mathbf{w})\|_{2} \geqslant r_{1}$, so that in either case

$$
\left\|\mathbf{w}^{*}\right\|_{c}=\left\|\mathbf{v}_{i}^{*}-\mathbf{v}_{j}^{*}\right\|_{c} \geqslant r_{0} .
$$

For $\mathbf{x}^{*} \in \mathbf{W}$ let

$$
B\left(\mathbf{x}^{*}, \lambda\right)=\left\{\mathbf{y}^{*} \in W:\left\|\mathbf{x}^{*}-\mathbf{y}^{*}\right\|_{c}<\lambda\right\} .
$$

Now consider the $7^{m}+1$ balls $B\left(\mathrm{v}_{k+i}^{*}, \frac{1}{2} r_{0}\right)$ for $0 \leqslant i \leqslant 7^{m}$. The triangle inequality and (3.4) show these are disjoint sets. Hence they occupy a volume $\left(7^{m}+1\right) V$ where $V$ is the volume of $B\left(0, \frac{1}{2} r_{0}\right)$. But by (3.3) all these balls sit inside the ball $B\left(0, \frac{7}{2} r_{0}\right)$, which has volume $7^{m} V$. This contradiction proves Theorem 1.2 .

\section{References}

[1] T. Cusick, 'Best approximations to ternary linear forms', J. Reine Angew. Math. 315 (1980), $40-52$.

[2] E. Dubois, 'Best approximations of zero by a cubic linear form. Calculations of the fundamental unit of a not totally real cubic field', Proc. of the Queen's Univ. Number Theory Conference, 1979, edited by P. Ribenboim, pp. 205-222 (Queen's Papers in Pure and Applied Mathematics No. 54 (1980)).

[3] E. Dubois and G. Rhin, 'Approximation simultanées de deux nombres réels', Séminaire Delange-Pisot-Poitou, 20e année 1978/1979, Théorie des Nombres, Fasc. 1, Exp. 9, (Secrétariat Math., Paris, 1980).

[4] E. Dubois and G. Rhin, 'Meilleures approximations d'une forme linéaire cubique', Acta Arith. 40 (1982), 197-208.

[5] W. B. Jurkat, W. Kratz and A. Peyerimhoff, 'On best two dimensional Dirichlet approximations and their algorithmic calculation', Math. Ann. 244 (1979), 1-32.

[6] J. C. Lagarias, 'Some new results in simultaneous Diophantine approximation', Proc. of the Queen's Univ. Number Theory Conference, 1979, edited by P. Ribenboim, pp. 453-474 (Queen's Papers in Pure and Applied Mathematics No. 54 (1980)). 
[7] J. C. Lagarias, 'Best simultaneous Diophantine approximations. I. Growth rate of best approximation denominators', Trans. Amer. Math. Soc. 272 (1982), 545-554.

[8] H. Williams, G. Cormack and E. Seah, 'Calculation of the regulator of a pure cubic field', Math. Comp. 34 (1980), 567-611.

\section{Bell Laboratories}

Murray Hill, New Jersey 07974

U.S.A. 